\title{
The Problem of Time
}

\section{Li Choy Chong, Luisella Balestra}

Asia Research Center, University of St. Gallen, St. Gallen, Switzerland

Email: Li-Choy.Chong@unisg.ch

Received 13 February 2014; revised 13 March 2014; accepted 20 March 2014

Copyright (C) 2014 by authors and Scientific Research Publishing Inc.

This work is licensed under the Creative Commons Attribution International License (CC BY).

http://creativecommons.org/licenses/by/4.0/

\section{Open Access}

\begin{abstract}
Time is a difficult topic for the scientist. This paper investigates how this particular subject is considered in economics today and what the implications are. We demonstrate that today orthodoxy has avoided to treat the temporal element, creating a dangerous contradiction at its inside. A reconsideration of time has to be formulated, since the reintegration of the chronological entity offers better interpretations for several phenomena such as economic crisis.
\end{abstract}

\section{Keywords}

Time; Economic Modelling; Economic Dynamics

\section{Introduction}

This paper would like to discuss two anomalies that mainstream economics maintains at its most basic level. These congenital incongruities, indeed a part of today fundamental dogmas, are responsible for many of the limits of economic orthodoxy and are caused by an incorrect treatment of a particular and theoretically difficult element, time. With the term "mainstream economics" (and economic orthodoxy and other similar expressions), we are referring very broadly to the whole body of economic theories, whose methodology is based upon the one that Paul Samuelson derived and formalized in his Foundations of Economic Analysis and whose main characteristics are the concept of economic system as a system in equilibrium and the hypotheses of maximisation and stability, as fundamental instruments to define main variables and theorems ${ }^{1}$.

The temporal entity is a true enigma that neither scientists (from any field) nor philosophers even know how to define exactly. In fact, it is virtually impossible to give one simple explanation of this term, without becoming trivial ("Time is a collection of instant"), too imprecise ("Time is the dimension of the causality") or cryptic

\footnotetext{
${ }^{1}$ In more recent years, we have to consider also Robert Lucas, Nancy Stokey and Thomas Sargent with the dynamic programming methods (see later in the paper for an example taken from Lucas). We have decided to use a methodological criterion to define theories instead of one based upon the content parts. This is due to the fact that the time-problem is inherent to the primordial core of any economic theory and therefore the methodological can be much more useful for differentiating. Moreover, this paper is concerned with a matter of method (the use of the time and the formalization of this use in economics), consequently a methodological selective mechanism is more appropriate.
} 
("Time is the flow of events past the stationary I"). To broadly characterize it, we have to rely on some background theories (“Does time really exist? How is time related to time? Is the future real?”), but by doing this we must consider an enormous range of arguments and positions that philosophers and scientists have been discussing for 2500 years, in several cases with a lot of questions still left unresolved. To get a glimpse of this huge and multivariate debate, first we can remember the dispute about the effective existence of time, whether it does exist or not independently from the events occurring at its inside. This problem, discussed since Plato and Aristotle, has implied basically two points of view: the first, called relationism with respect to time ${ }^{2}$, argues that the temporal concept is so strictly connected to the idea of motion and variation that without change therefore events there would be no time at all; the other perspective, named Platonism, has insisted in comparing time with a container and therefore admitting its existence outside the facts occurring in it ${ }^{3}$. Another crucial problem discussed around the temporal topic has been the link between time and mind (discussed by St. Augustine and Aristotle) from which several further elements, debates and arguments have been derived. Among them, there are the distinctions between psychological and physical time, the perception of time and its duration, the flow of time and the formulation of the entropy concept, the specification of the four-dimensional space-time, and the fatalism (the list can be much longer!)

In front of this huge conceptual flourishing, the economic science has maintained a highly unsatisfactory attitude. In fact, economics has turned out to be almost indifferent to the richness of implications and meanings of the disputes around the chronological topic ${ }^{4}$, and when the time element is considered, it is scarcely accepted as a relevant element and basically dismissed as soon as possible from the cognitive elaboration (on a formalization level) despite its crucial importance (both empirical and theoretical). The problem of mainstream theories is determined not only by their inability in recognising the importance of the chronological element, but also by the difficulty in getting its complexity incorporated without excessive simplification. This inadequate dealing of such a hard subject is responsible for the problems in comprehending several economic phenomena (mostly those characterised by change such as economic crisis) and can be ascribed for the lack of realism that many "new theories" (such as behavioural economics and new institutional economics) criticize to the orthodoxy. In fact, how can economic models represent reality if they forget such a relevant part of it? Aim of the present paper is to illustrate this "strange" attitude in today economic orthodoxy as well as its implications. First, we will delineate the empirical significance of time. Secondly, we will observe the uneasy treatment it receives on a theoretical level, as, mainstream theory, on one side, is built upon this concept and on the other, denies explicitly its significance and in this way determines an extremely confused situation right at the heart of several contemporary theories and methodologies. To conclude this introduction, we would like to offer our definition of time, despite all the limits this definition might have, as well as an important caveat for our paper. "Time passing" is a complex of countless multitudes of interrelated variables that interact with the observed economic variable and caused it to change over time. This complex of "time passing" consists of the spatial arrangement of interacting variables which we normally refer to as the "context" in a specific or fixed moment, which changes in its configuration through some intervening variables in time, thus changing the "context", and with the variable under observation. In time passing, one observes economic behavior over time as well as changes in economic variables. Clearly we are taking into account an element of high complexity, whose analysis necessarily implies some sort of simplification but that cannot be excluded or ignored. One of its peculiarities is the immanent character, namely the fact that time is everywhere and everything that can be considered under this point of view. Thus, it is very difficult to delimitate time as a subject inside economic theory and therefore, for a paper discussing this kind of topic, there's a high risk of becoming too generic, with insufficient and/or arbitrary arguments ${ }^{5}$. To face this problem, we have decided to try to be focused on definite elements, namely business cycles evidences for the empirical section and the treatment of time (primarily) by the most important macroeconomics schools of thought for what it concerns the theoretical side. Despite all of this, we are aware that the risk of an excessive generalization or insufficient argumentation might remain still strong, probably due to the particular element we are dealing with; it might be impossible to get it to "normal" level. Nevertheless we think it is very useful to concentrate our analysis on this too long ignored element, it's definitely time to do it. How to treat it specifically

\footnotetext{
${ }^{2}$ Aristotle and Leibnitz are some of the philosophers adopting this point of view.

${ }^{3}$ Plato, Kant and Newton can be considered as some of the sustainers of this point of view.

${ }^{4}$ Notice that we are not saying that the time has been ignored, but simply that it has been only marginally considered for its implication. Its complexity has been forgotten.

${ }^{5}$ Since our purpose is to offer a general overview about the uses (and abuses) of the temporal element in economics and not on delimited points, for us the danger is even higher!
} 
is more than that we are intended to deal with here, and our purpose is to make the case for such a debate open.

\section{Time in Economics-The Empirical Relevance}

We can look at two different kinds of evidences to observe the empirical importance of the time passing in economics (although we don't have to forget that the risk of excessive generalization is particularly strong in this section):

- papers supporting the presence of random walk for business cycles

- papers supporting non-linear economic structures

The first source relates mainly to the papers ${ }^{6}$ showing the presence of a random walk in the business cycles: from the study of Nelson and Plosser, to the works, among others, of Campbell and Mankiw Stock and Watson and Durlauf and so on. Fundamentally, what is remarkable for us among them is the impossibility of rejecting the hypothesis that the data for economics in time can be time-dependent series with no tendency to return to a predetermined trend line. In other words, these works, that we won't review here, point to the chronological nature of the economy: what is happening at $t_{1}$ can influence the output in the future $t_{2}$ in an irreversible way. Consequently, a relationship between time and economics that might even be complex, unpredictable and not deterministic cannot be excluded and surely can be hardly represented by some always—valid (and therefore out of time) rule, as economic theories would typically suggest instead.

Moreover, according to the above facts, another very common device used by mainstream theories seems to suffer for the lack of empirical support: the use of external shocks for justifying changes of situation. In fact the assumption of an economic system only randomly perturbed and otherwise eternally tied up to the same theoretical schemes matches only partially with the observation that economics is time-dependent and therefore the variations can be permanent and part of its dynamic. The second kind of support about time and economics comes from the area of non-linear economics, although the sources of evidences are quite fragmented and diversified. In fact, whereas the presence of nonlinearities by macroeconomic data seems to be a well-documented fact, the empirical confirmations about non-linear models are not so clearly outlined especially in case of chaos. Paradigmatic in this sense is the work of Brock and Sayers of 1988, one of the first papers devoted to the detection of chaotic behaviour; since it expresses an ambiguity about the results that even today remains typical for this kind of paper. In fact the two authors observe little evidence for chaos, nevertheless they cannot exclude that this outcome can be determined by weakness of the tests employed. In later years, the paper of David Harvie [1] examines Richard Goodwin's non-linear growth cycle model. Goodwin's cycle is an extremely simple model, employing only two factors of production, capital and labour. The model describes a closed cycle, like the ones in the Figure 1 . With $\left(\mathrm{u}^{*}, \mathrm{v}^{*}\right)$ as central points. Its mechanics can be understood by considering a point $\mathrm{Y}$, where $\mathrm{u}>\mathrm{u}^{*}$. If employment is high, labour is in a strong position to press for higher wages; necessarily profit will fall, thus squeezing investment. Low investment will reduce employment rate, tilting the balance of power in favour of capitalists. High profits will again raise investment and eventually the employment rate as well, at a later moment. Given the equilibrium point $\left(\mathrm{u}^{*}, \mathrm{v}^{*}\right)$, the trajectory described by the arrow depends only on the initial conditions. In case of perturbation (for example a stochastic shock), the path will continue in a different cycle (for example, in the figure above, there can be a switch from the bigger to the smaller cycle) and no forces can bring it back to the original position. The model does not have any interpretation or suggestion for the economy's starting point and any reason for the variables fluctuating at different levels. However it does imply a chronological irreversibility that can be of interest for us. Its empirical assessment can be developed in 2 ways:

- Goodwin's model suggests a dynamic relationship between capital and work that can be evaluated through trajectories with actual data

- we can compare the actual data with the ones predicted by the model

Harvie uses the data for ten OECD countries and computed first the uv trajectory, and then he compared the results predicted by Goodwin formulas with the effective ones ${ }^{7}$. These are his conclusions about the econometric predictors (quantitative analysis):

"Our results about the simple Goodwin model tested here are mixed, though perhaps not unexpected. First, our predictions of the growth cycles centre $\left(u^{*}, v^{*}\right)$ and periods $T$, based upon estimates of the five parameters $\alpha$, $\beta, \sigma, \gamma$ and $\rho$ are inaccurate. Second, Goodwin's neglect of monetary considerations in his earnings/Philips

${ }^{6}$ Obviously the ones above aren't the only works useful for proving the relevance of time. In fact, due to its peculiar characteristics, “time is everywhere" and therefore evidences of its importance can be found in many other ways.

${ }^{7}$ This comparison can be done by employing the fact that the central values of the cycle in Goodwin's formula are their mean as well. 
curve equation is not warranted, nor is his assumption of capital-output ratio constancy. But, given our findings that the simplification cannot be justified, one would not expect predictions based upon this model's parameters to yield promising results. Indeed given the very simple nature of the model, it would be more puzzling the opposite, were it to fit the data well."

However, when he comes to the qualitative analysis, Harvie's conclusions are different:

"The evidence presented in Figure 2 lends broad qualitative support to Goodwin's model. For all ten countries being investigated, the phase portraits shows clearly that, concentrating on the 'big picture', a 'high' employment rate is followed by a rising share of national income accruing to workers; this is followed by employment; this in its turn, seems to lead to the diminishing of workers' once more."

In other words, the temporal irreversibility implied by Goodwin's model cannot be rejected by empirical data and therefore a complex link between time and economics cannot be excluded. On the whole, if we concentrate on the big picture, empirical researches in the non-linear economics area confirm a more relevant role for the time element than the one usually assumed by theory, although the models employed don't seem particularly efficient in representing economic facts.

Thus, to conclude this first part, we observe the impossibility of rejecting the hypothesis that the data are timedependent series with no tendency to return to a trend line. From our point of view, this means that we fail to

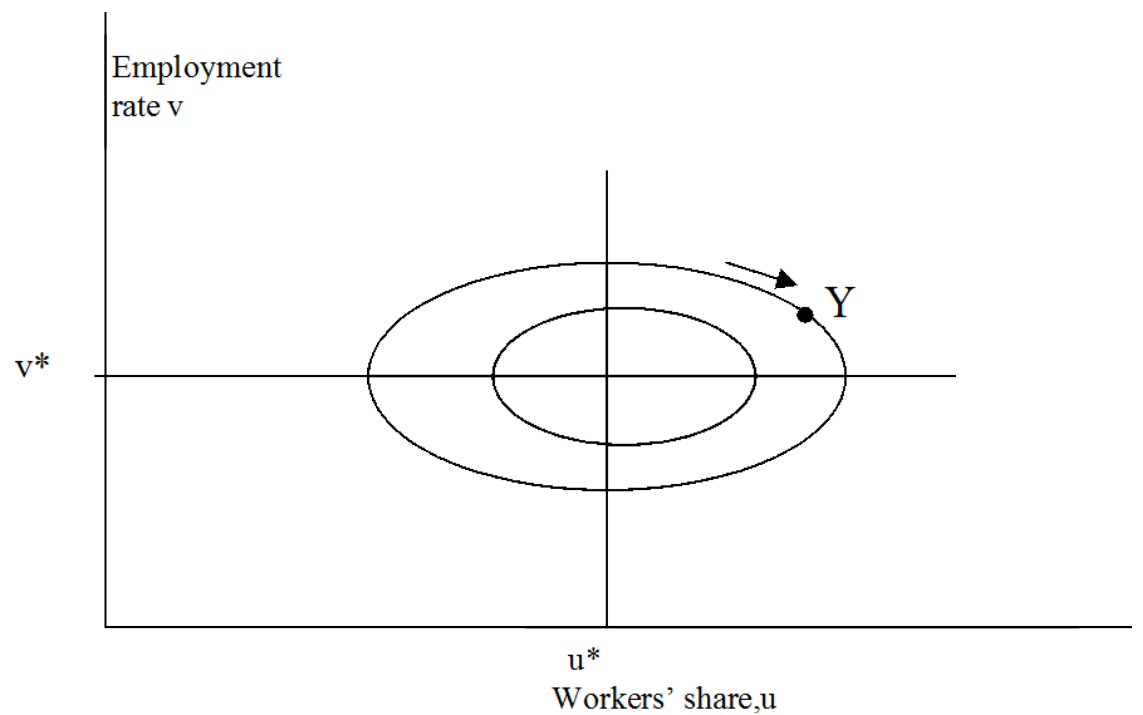

Figure 1. Goodwin's cycle model.

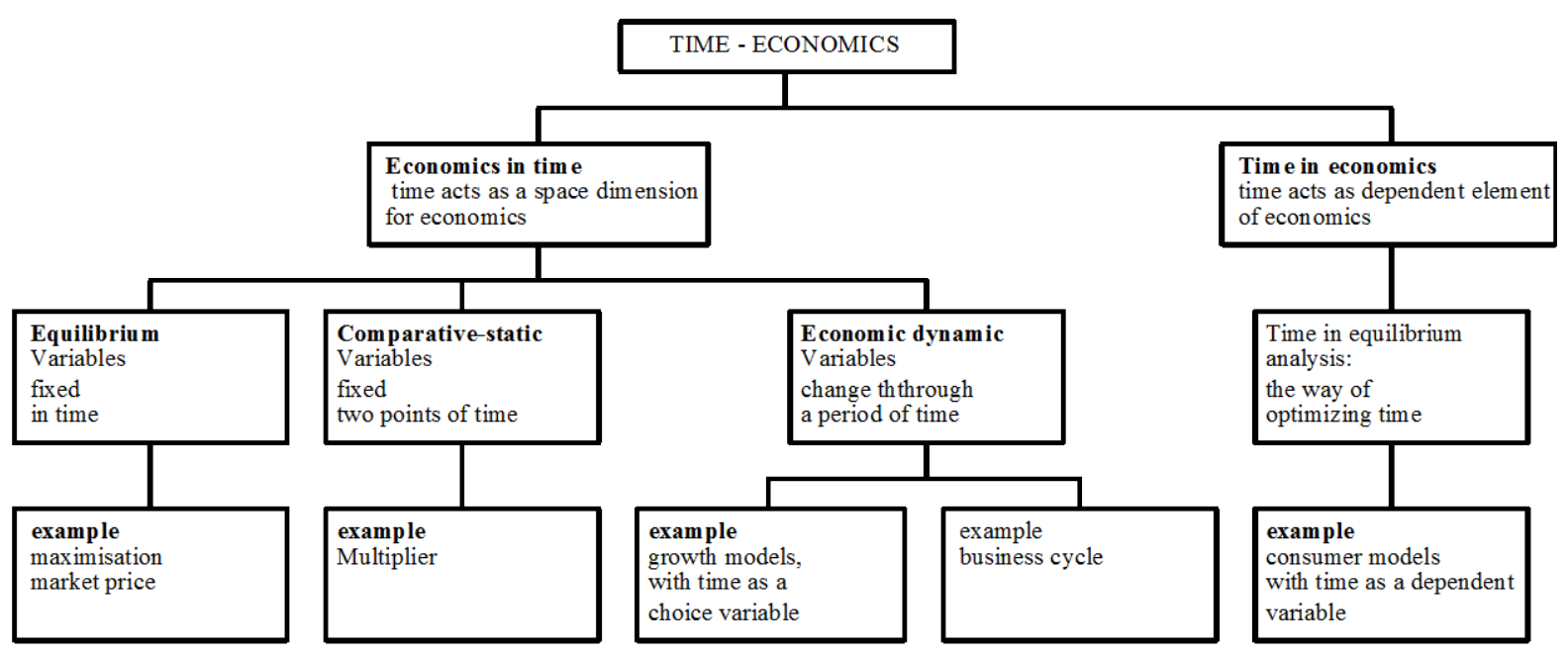

Figure 2. The role's of time in economic theory. 
reject empirically a complex relation between time and economics and therefore the theoretical analysis should be aware of its existence, trying to deal with despite its existence. As we will see in the next session this requirement is highly unattended.

\section{Time in Theory}

We have learned by the previous section that a (complex) link between time and economic system finds some support from an empirical point of view. This would suggest at least some awareness in the theoretical treatment of the economic phenomenon, about the role of the temporal element. Nevertheless, quite surprisingly, not only common theory fails recognizing time as relevant for economics, but also bases its methodology upon its explicit denial. To get an idea of this "strange" situation consider the following example proposed by Lucas [2] that expresses a common practice and aptitude for dealing with the chronological element. Here, a social planner must maximise the following utility function of a representative household, which is supposed to live infinitely:

$$
\begin{gathered}
\max \sum_{t=0}^{\infty} \beta^{t} U\left[\left(c_{t}\right)\right]=\beta^{t} U\left[f\left(k_{t}\right)-\left(k_{t+1}\right)\right] \\
\text { s.t. } 0 \leq k_{t+1} \leq f\left(k_{t}\right) \\
k_{0}>\text { given } \\
c_{t}=\text { consumption at time } t \\
U\left[\left(c_{t}\right)\right]=\text { utility function of consumption at time } t \\
\left(k_{t}\right)=\text { capital at time } t
\end{gathered}
$$

$$
f\left(k_{t}\right)=\text { total supply of available goods at time } t \text { per worker including undepreciated capital }
$$

The solution can be found according to the following, first order and boundary conditions ${ }^{8}$ :

$$
\begin{gathered}
\beta f^{\prime}\left(k_{t}\right) U^{\prime}\left[f\left(k_{t}-k_{t+1}\right)\right]=U^{\prime}\left[f\left(k_{t-1}\right)-k_{t}\right] \\
k_{T+1}=0
\end{gathered}
$$

In the definition of the economic problem as well as its solution, time represents simply a space dimension and has nothing to do with the economic mechanism of interest that can be transformed from a future into an infinitely repeated present one as a result. In both cases, the chronological element is formally irrelevant but, contemporaneously, it is also the necessary condition for the recursive formalization of the economic dilemma. In fact, it is thanks to the uninfluential and fixed temporal tenses that the economic behaviour of interest can be identified and this condition cannot be changed in later moments, unless some sort of external random shocks that modify this immutable position are supposed. Otherwise, time (formally) elapses without affecting anything and can consequently be eliminated. In effect, Lucas eventually achieves the complete and explicit removal of the temporal element, reduced to a mere index differentiating variables. In his book, Lucas speaks expressly of nuisance, but he is inverting the causal order between recursive approach and the unimportance of time!

"Notice that when the problem is looked at in this recursive way, the time subscript have become a nuisance."

The example evidences the confused attitude of today mainstream doctrines toward time: on one side (creating the theory) time plays a crucial role, significantly shaping the economic elements, on the other (formalizing the theory) time plays only a negligible part within the maximisation framework and has no role as independent variable. Such a kind of result implies the following:

- the recursive hypothesis suggests an economic behaviour absolutely fixed, unidirectional and completely disconnected with respect to the surrounding environment. Even in case of external shocks it presumes that the momentum (the continual motion after a movement) persists constantly the same

- we are in the impossibility of explaining the concept of change within the economic theory (being excluded from its fundamental mechanism), we have to assume the external random-shock mechanism

- theory is timeless, while the empirical data used to test its soundness are grounded on time

- the solution to be insightful usually requires several particular mathematical simplifications (such as concavity and additive separable form for the objective function). Thus it can't be considered a universal technique

\footnotetext{
${ }_{8}^{8}$ For simplicity Lucas change the problem from infinite to a T horizon problem.
} 
to adopt, we have to be careful in identifying the necessary assumptions

The first and second implications are of particular interest, because they represent the main severe limits for economic orthodoxy and its representation of time as a space dimension: the management of time passing (and economics changing) can be done only by supposing the economic mechanism completely impermeable to what happens at its outside and the mutation is allowed only through the device of the external shock, this is to say on an exceptional rather than regular basis. In fact, first, economics is imagined in a timeless situation of equilibrium, then, some external factor is supposed to disturb this situation. Thereafter, it is assumed that the economy would return to another stable position. In this way, the wide theme "variations in economics" is reduced to a mechanical schema "impulse -> reaction", which is too limited, given the complex reality of multiple constantly interacting variables in an economic context. Clearly, all these temporal-required suppositions are particularly strong, arising consequently doubts about the ability of the models in representing the reality (they fail to embody effectively such a relevant part of it!). In particular, they are formulated with an infinite horizon and the expectation operator, for this second represents the way individual faces the ignorance of future: it's part of his perceptions and evidently can't be conceived completely fixed and immovable, eternally insensitive to the influences of the surrounding environment. This time-anomaly inside mainstream theories is widely extended and deeply inserted. To observe this fact consider the Figure 2 representing the ways economics and time are conceived to interact together and that contains virtually all the main topics usually treated and analysed by economic theory. There are two possible ways of associations: with economics time can act as a mathematical space dimension or as its dependent element, in both situations being without definition by itself. By the first case, the most extended, economics becomes "economics in time", that is through the combination with the chronological element the system assumes its temporal collocation and since we have different kind of times, we can have different kinds of "economics in time", at least three:

- economic equilibrium (economics in one single instant of time);

- comparative-static (economics of two or more moments);

- economic dynamics (how an economics of the single moment evolves into another one). ${ }^{9}$

Considering this point of view, we can say that basically all contemporaneous economic doctrines are economics in time, as basically their whole theoretical structure can be defined or inserted in one of the categories seen above. To see this element we can look at the third line of the scheme and notice the thematic variety of the quoted examples as well as their extreme widely employment.

The second branch of the figure represents the other case to be observed, when time becomes part of economics (time in economics). Here the role of the temporal entity is very limited, it has to be viewed as the result of an economic mechanism (being the dependent element) and therefore not as its active factor ${ }^{10}$ and once again the third line shows us some possible illustrations of this different use of the temporal component. The exclusion of time from the theoretical thinking derives from several elements. First, by the fact that the chronological phenomenon represents mostly a space dimension and not an element by itself: being a space dimension implies in effect that time is simply a medium where other forms and structures are realized, absolutely kept out from their functioning. Moreover, if we compare the interpretation offered by economic science to the richness of meanings and implications that other disciplines have been able to derive from the time concept, no doubt that the chronological phenomenon is poorly represented in this situation since many of its features, such as the distinction between objective and subjective time and its irreversibility, are excluded, thus determining the highly ineffectiveness of the space dimension characterization. A further element that shows us the exclusion of time from the relevant mechanism can be identified in the equilibrium assumption for the economic system. This position, which provides the logical basis for all the categories of economics in time (and therefore for mainstream theories), involves a complete independence from the time passing and such condition, holding the temporal factor invariant, eventually allows for its formal complete elimination as seen before. Nevertheless, despite this formal exclusion, the temporal element still maintains its "shaping ability" in defining theoretical elements of interests. In fact the relation with time (invariant) is the main feature of the equilibrium concept (variable are in equilibrium = they won't change anymore = they are independent from time), which, on its turn, provides the logical basis for the economic theorizing (equilibrium, comparative-static, dynamic analysis). Thus, just as in

\footnotetext{
${ }_{9}^{\bar{B} \text { Basically all contemporaneous economic doctrines }}$ are economics in time, as basically their whole theoretical structure can be defined or inserted in one of economics in time categories seen above (third line of the scheme).

${ }^{10}$ Consider further that time as dependent element is usually treated as variable during optimization processes, which in turn can be considered as a particular subset of equilibrium case.
} 
Lucas' example, time though being formally expelled defines theory crucially. Moreover this anomaly with its incongruence and weakness is deeply inserted at the basis of main theories, being so deeply part of their methodology.

Two of the most famous non-orthodox doctrines, the Austrian and the Post Keynesian, have both noticed the inappropriate use of the temporal element inside mainstream theory. In particular they have done it with respect to the concepts of equilibrium, knowledge and agent's planning. In fact, before being able to act concretely, the individual must do some plans and for this he must use his knowledge, which evolves continuously as time goes by. Consequently, equilibrium can be understood only as compatibility between different plans of each individual composing the society, it is extremely weak as analysis tool for the economic reality and, most of all, it depends on time completely: a timeless equilibrium is a meaningless equilibrium. In the words of Hayek [3]:

“(...) It is obvious that the passage of time is essential to give the concept of equilibrium any meaning. (...) Many economists appear to have been unable to find a place for time in equilibrium analysis and consequently have suggested that equilibrium must be conceived as timeless. This seems to me to be a meaningless statement."

And in later years the words of O'Driscoll and Rizzo [4]:

"In a world of time and genuine uncertainty, there is ceaseless endogenous change (...) Equilibrium has traditionally been centred on the absence of endogenous change. It has been conceived as the state of affairs produced after all the endogenous forces have been worked out. From this perspective, time and equilibrium are surely incompatible."

"As long as time is allowed to pass, knowledge will grow and there will be endogenously produced change. Accordingly, it is not possible for the system to settle down to an equilibrium whether of the exact or stochastic variety."

Nevertheless, though being able of recognizing the time problem, Austrian economists fail to offer a more effective solution to it. What is missing by them is the ability of completely deriving the consequences of their recognition of the time problem and get them inserted inside their theoretical view. In fact, even here time remains a space dimension of the economic phenomenon, just as by mainstream economics. The only difference is the admission of an indirect effect of time on economics through individual knowledge or capital goods. See for example Roger W. Garrison [5]:

"While there is no market for time as such, the time element is inextricably wedded to every market that does exist. The analysis of a market economy consists of identifying actions of individuals that give rise to various market phenomena, and time, literally, is the medium through which these actions transpire."

Given the above, it is clear that the passing of time, although important, represents once again, only a dimension in the system, with the individual's knowledge being its crucial element. This being the case, Austrian (Post Keynesians) schools have themselves committed the same error in neglecting the multiple and interacting effects of time passing, as the position of temporal element and the consequent structure of the economic reasoning remain the same as that of mainstream schools.

\section{Conclusions}

In this paper we have evidenced a crucial anomaly that mainstream economics maintains its fundamentals. This situation is connected to the difficulties that today orthodoxy has in dealing with the time problem. In effect, the handling of this subject, with its immanent complexity, has shown two serious weaknesses: the first caused by the difference between its empirical and theoretical treatments (the latter is unable to express the link between time and economics that the former evidences); the second determined by the contraposition among the structures of economic theorization (based upon the concept of equilibrium and consequently based upon time) on one side, and its formal representation on the other (characterized by the denial of the temporal entity). The fact that economics is something that cannot remain constant during the passing of time is totally accepted and recognised in theory and in practice. We may, for example, refer to the widely used techniques for evaluating (generally) money in the future: the interest rate tool and the discounting value. The use of these tools would testify the fact that economics does not remain the same with time passing and the practical usefulness of economic perspectives with time incorporated. Nevertheless, economic theory seems to "forget" that time is a relevant element. In effect the temporal factor is always present in every structure (cannot be excluded), and is responsible for some of its basic characteristics (such as expectations) and for every different ways to conceive it. There is a different way to conceive economics. Practically every economist, when developing his own theory, has to face a veritable time problem, which could not be ignored, and since paying no attention, it will inevitably lead 
to a dramatic loss of realism in the conjectural model. Paradigmatic in this sense is the case of mainstream economics that has adopted a highly unsatisfactory way to deal with temporality. This incoherent position has led to several drawbacks: first the loss of power for this kind of models as a tool in representing human reality, missing such important element; second the inability to explain the concept of change, uneasily inserted in a mechanical scheme of exogenous shocks; third the serious difficulty in representing the time component adequately, too simply expressed by a projection of economics into an infinite temporal horizon which transcends the single instant and where restrictive assumptions are required to keep it reasonable.

Quite surprisingly, this deficient situation about the temporal factor seems to be a common element with nonlinear economics. Though not among the aims of this paper of a discussion around this theme, the stress is in the adoption of non-linear mathematical techniques because of its better representativeness of economic reality. But this approach, based after all on similarity, shares the same philosophical view as the orthodox one, namely the complete assimilation of the human being to a scientific element and the consequent inattention to time as a human fact as well as the oblivion of its multiple meanings. From this point of view, the results are equally unsatisfying: we might have better models in reproducing human reality, (but this remains an open question) but we still lack an explanation able to comprehend such a relevant element for our lives (time). The drawbacks are not very different from the ones seen before, whereas before the event of change, they were represented by a random external shock occurring to the economics; here the unpredictability is simply conglobated into the economic system through the assumption of nonlinearity. But once again the reasons of the mutation (where time would play surely an important role) stay outside the possibility of explanations of the model, just as before.

The discovery of this time anomaly in today economic mainstream is of high interest, as it gives new perspectives in several economic fields. For example, implications can be extremely relevant with economic dynamics. Here the choice of a chronological concept more appropriated than one conceptually based upon the standstill of equilibrium can become vital. We may even identify a new notion for economic theory, namely the speed of the economic elements. By "speed", we mean the degree and the kind of interaction between economic variables on one side, and time on the other. We think the specification of this element, as a basic fundamental of economic theory, is essential, since it allows obtaining a more powerful analysis of economics than the one based upon the concept of equilibrium. The idea of a new consideration of time in economics can also have implications for some of the most relevant debates in economic literatures, such as those around business cycle and growth theory. In these cases, would it not be possible to assume that economics changes by itself ?

What will be the consequences of this new time element in these debates? At first sight, at least, whether variations come on the supply rather than on the demand side would become fundamentally irrelevant. But a new time awareness would also offer additional new policy instruments for modifying the economic mechanism, by speeding up or slowing down the temporal movements of some chosen variables, particularly in the case of economic crises. For example, capital controls to stop the outflow of capital in a country could be seen as an attempt to arrest the rapidly deteriorating economic circumstances and to revert these unhealthy circumstances over time-effectively leading to a re-alignment in the speed of different economic variables. Or, in more general terms, a temporal realignment between different economic agents is a guiding policy to be pursued by a government in case of economic crisis or panic.

Our results have been demonstrated only on a methodological basis and clearly there's a need for further investigations, nevertheless we hope that they can serve as source of evidence for what it concerns the limit of today economic orthodoxy (in this sense there is a link to the growing literature that criticizes mainstream economics for its lack of realistic assumptions-e.g. behavioural economics). We have demonstrated the basis for the work to be done in the future, namely that today mainstream doctrines have a congenital anomaly determined by a "wrong" relation with the temporal entity that automatically limits the effectiveness of their results. There is still a long way to go to obtain more substantial results from a solution to the time problem, but we think that this can be a fruitful operation to develop in the future.

\section{References}

[1] Harvie, D. (2000) Testing Goodwin: Growth Cycles in Ten OECD Countries. Cambridge Journal of Economics, 24, 349-376. http://dx.doi.org/10.1093/cje/24.3.349

[2] Lucas, R.E. and Stokey, N.L. (2001) Recursive Methods in Economic Dynamics. Harvard University Press, Cambridge, $10-11$. 
[3] Hayek, F.A. (1937) Economics and Knowledge. Economica, 4, 33-54. http://dx.doi.org/10.2307/2548786

[4] O’Driscoll, G.P. and Rizzo, M. (1996) The Economics of Time and Ignorance. Routledge, London.

[5] Garrison, R.W. (1984) Time and Money: The Universal of Macroeconomic Theorizing. Journal of Mac Roeconomics, 6, 197-213. http://dx.doi.org/10.1016/0164-0704(84)90005-3 